\title{
Development of a Risk Predictive Scoring System for Epilepsy in Infants with Paroxysmal Motor Events: A Retrospective Single-Center Study
}

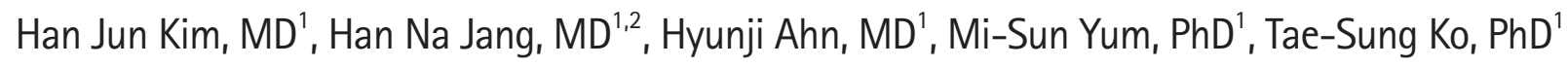 \\ 'Department of Pediatrics, Asan Medical Center Children's Hospital, University of Ulsan College of Medicine, Seoul, Korea \\ ${ }^{2}$ Department of Pediatrics, College of Medicine, Kyung Hee University, Seoul, Korea
}

Received: September 21, 2021

Revised: November 12, 2021

Accepted: October 17, 2021

Corresponding author:

Mi-Sun Yum, PhD

Department of Pediatrics, Asan

Medical Center Children's Hospital,

University of Ulsan College of

Medicine, 88 Olympic-ro 43-gil,

Songpa-gu, Seoul 05505, Korea

Tel: + 82-2-3010-3381

Fax: +82-2-473-3725

E-mail:yumyum99@hanmail.net
Purpose: Paroxysmal motor events are common clinical symptoms in infants visiting pediatric neurology clinics. Due to the heterogeneous clinical symptoms and the difficulty of interpreting electroencephalography (EEG) in infants, differentiating paroxysmal motor events from epileptic events is challenging. This study aimed to investigate the risk factors for the diagnosis of epilepsy in infants and to develop a scoring system that predicts the risk of epilepsy.

Methods: We retrospectively analyzed data from patients who presented with paroxysmal motor events in infancy between January 2008 and December 2009 at Asan Medical Center. Electronic medical records were reviewed for patients' demographics, medical history, clinical characteristics associated with specific situations, and motor symptoms. Laboratory findings, EEG, and brain magnetic resonance imaging were also reviewed.

Results: In total, 111 infants with paroxysmal motor events were enrolled. Non-epileptic paroxysmal motor events (NEPMs) were associated with specific situations $(P<0.001)$. Patients with epilepsy were likely to have focal motor symptoms $(P=0.08)$, a medical history of a neurologic disorder, and/or a family history of epilepsy $(P<0.05)$. A risk scoring system was developed based on these risk factors; using this system, infants with 2 or more points could be diagnosed with epilepsy with $61.76 \%$ sensitivity and $88.31 \%$ specificity.

Conclusion: Infants with paroxysmal motor events were more likely to be diagnosed with NEPMs than with epilepsy. An absence of specific situations for paroxysmal events, focal motor seizures, and a medical history of another illness were associated with the final diagnosis of epilepsy.

Keywords: Seizures; Epilepsy; Infant; Risk factors

\section{Introduction}

Non-epileptic paroxysmal motor events (NEPMs) are episodes of paroxysmal changes including behavior, sensation, or consciousness that lead to repeated, intermittent, and unusual movements within the same context [1-3]. Since it is difficult to differentiate epileptic seizures from NEPMs, the most helpful approach is to obtain an informative history from an eyewitness combined with a home-video recording. In contrast, epileptic seizures are defined as recurrent, unprovoked events due to abnormally excessive neuronal firing in the cerebral cortex. Epileptic seizures need to be treated with an antiepileptic drug and may be associated with other

Copyright (C) 2022 Korean Child Neurology Society

This is an Open Access article distributed under the terms of the Creative Commons Attribution Non-Commercial License (http://creativecommons.org/licenses/by-nc/4.0/) which permits unrestricted non-commercial use, distribution, and reproduction in any medium, provided the original work is properly cited. 
childhood neurocognitive disorders [4]. According to recent studies, in pediatric patients admitted with paroxysmal events suggestive of seizure, $24 \%$ to $47 \%$ of children were diagnosed with NEPMs, whereas childhood epilepsy was diagnosed in $4 \%$ to $10 \%[5,6]$.

To optimize management and to guide parents regarding the patient's prognosis, it is important to establish the correct diagnosis through a detailed medical history from the patient and primary witnesses as well as careful observations. However, differentiating paroxysmal motor events from epileptic events is challenging due to the heterogeneous clinical symptoms and the difficulty in interpreting routine interictal electroencephalography (EEG) in infants. Video EEG is necessary for the complete characterization of an event as nonepileptic; however, the application of video EEG monitoring is difficult due to various factors, such as the limited number of facilities, high cost, and technical challenges.

In addition, previous studies on the diagnostic approach after paroxysmal motor events nearly focused on diagnostic tools, including the video EEG findings of specific NEPMs, due to their heterogeneous etiologies [1-3,7-9]. Other studies have made efforts to identify clinical variables that aid in the diagnosis, such as motor symptoms, demographic findings, medical history, and stimulation at motor events as a predictor of the diagnosis of NEPMs $[10,11]$, but the clinical variables in those studies were not standardized. Thus, there is a crucial clinical need for a standardized scoring system that would facilitate the differential diagnosis of epilepsy and NEPMs.

In this study, the clinical risk factors of epilepsy in infants with paroxysmal motor events were investigated, and we further attempted to develop a scoring system to identify infants with a high likelihood of having epilepsy.

\section{Materials and Methods}

\section{Patient enrollment}

This retrospective study was performed from January 2008 to December 2009 at Asan Medical Center, which is a tertiary referral center in South Korea. The study analyzed the electronic medical records of patients who presented with paroxysmal motor events in infancy ( $<12$ months), with changes in behavior, sensation, or consciousness that led to repeated, intermittent, unusual movements, and underwent EEG. Patients with a history of anti-epileptic drug use or epilepsy were excluded from the study.

For each patient, the final diagnosis of the paroxysmal motor event was classified as NEPMs or epilepsy based on a clinical assessment and a review of the investigations. The type of epilepsy was simply classified as focal, generalized, or unclassified. The
NEPMs were further classified as breath-holding spells, gastro-esophageal reflux, jitteriness, stereotypies, sleep myoclonus, tonic gaze deviation, shuddering, sleep behavior, the startle reflex, benign myoclonus, cyanotic spells, unclassified, or other.

Patients' medical records were reviewed to investigate the clinical data, laboratory findings, brain magnetic resonance imaging (MRI), and inter-ictal EEG findings. Demographic data were collected at the time of the first visit. The electrical medical records, which contained the patients' demographics, medical history, clinical characteristics associated with specific situations, and heterogeneous motor symptoms were reviewed by two pediatric neurologists. Specific paroxysmal motor event-associated situations, such as sleeping, feeding, crying or irritability, stimulation, and non-specific situations, were considered as clinical characteristics of the events.

In addition to patients' medical history and physical examination findings, routine EEG recordings obtained during hospitalization were reviewed. The presence of epileptiform activities or background abnormalities of the EEG were documented. Additional brain MRI scans and laboratory findings (electrolytes, glucose, and ammonia) were also reviewed.

This research was approved by the Medical Sciences Ethics Committee of Asan Medical Center (IRB file No. 2019-1287). Written informed consent by the patients was waived due to a retrospective nature of our study.

\section{Statistical analysis}

The data were analyzed using SPSS version 21.0 (IBM Corp., Armonk, NY, USA). The chi-square test was used to analyze qualitative variables, while mean values between the patients with epilepsy and NEPMs were compared using the independent t-test. Differences were considered significant at $P$ values less than 0.05 .

Univariate and multivariate logistic regression analyses were carried out to identify potential risk factors associated with epilepsy. A multivariate logistic regression model and the backward elimination method were used to estimate the odds ratios of epilepsy $(P<0.1)$ and to select the most important risk factors.

Based on the strongest risk factors, a scoring system for differentiating epileptic seizures and NEPMs was developed. We assigned a score using an estimate obtained by penalized maximum likelihood estimates using the Pearson's chi-square method. The total sum of each score was computed, and the optimal cut-off value was determined after calculating the sensitivity and specificity for different cut-off values. The performance of the scoring system was assessed using a receiver operating characteristic curve. 
Table 1. Demographic characteristics of the patients included in this study

\begin{tabular}{|c|c|c|c|}
\hline Characteristic & Epilepsy $(n=34)$ & NEPMs $(n=77)$ & $P$ value \\
\hline Sex & & & 0.12 \\
\hline Male & $23(67.6)$ & $40(51.9)$ & \\
\hline Female & $11(32.4)$ & $38(48.1)$ & \\
\hline History of prematurity (gestational age $<37$ weeks) & $4(11.8)$ & $13(16.9)$ & 0.49 \\
\hline Age of onset (mo) & $4.3 \pm 2.4$ & $4.8 \pm 3.0$ & 0.35 \\
\hline Age when EEG was performed (mo) & $5.2 \pm 2.2$ & $5.13 \pm 3.1$ & 0.93 \\
\hline Mean follow-up period (mo) & $54.2 \pm 48.5$ & $9.6 \pm 24.1$ & $<0.01$ \\
\hline
\end{tabular}

Values are presented as number (\%) or mean \pm standard deviation.

NEPM, non-epileptic paroxysmal motor event; EEG, electroencephalography.

\section{Results}

In total, 111 infants aged 1 to 11 months with paroxysmal motor events were enrolled. Of the 111 patients, 63 were male (57\%), and 48 infants were female (43\%). Thirty-four patients $(30.6 \%)$ had a final diagnosis of epilepsy, while 77 (69.4\%) patients were proven to have NEPMs. The mean age at the first paroxysmal motor event was $4.3 \pm 2.4$ months in the patients with epilepsy and $4.8 \pm 3.0$ in those with NEPMs. The demographic characteristics of the patients with epilepsy and NEPMs are summarized in Table 1.

No significant differences were found in demographic characteristics between patients with epilepsy and NEPMs except the follow-up period (Table 1). Within the epilepsy group, focal epilepsy occurred in 17 patients (50\%), and infantile spasms occurred in eight $(23.5 \%)$. Nine infants $(26.5 \%)$ were categorized as having unclassified epilepsy.

Table 2 shows the diagnoses of NEPMs in the 77 infants without epilepsy. Breath-holding spells $(n=13,16.9 \%)$ were the most frequent type of NEPM, followed by gastro-esophageal reflux, jitteriness, and stereotypies, while 13 NEPMs remained unclassified.

The laboratory findings of paroxysmal motor events were also reviewed as potential predictors of the diagnosis of epilepsy. Blood tests were performed only if clinically indicated, and no statistically significant differences were found between the infants with epileptic events and those with NEPMs (Table 3).

Fig. 1 shows the clinical characteristics of paroxysmal motor events. Compared to the infants with epilepsy, the patients with NEPMs were more likely to show associations with specific situations that preceded paroxysmal motor events, such as feeding, sleeping, crying, and some type of stimulation (72/77, $93.51 \%$ vs. $16 / 34,47.06 \%, P<0.001)$. Of these specific situations associated with NEPMs, situations related to sleep (e.g., falling asleep or arousal) were the most frequent, followed by crying or irritability, feeding, and others (Table 4).
Table 2. Final diagnoses of non-epileptic paroxysmal motor events

\begin{tabular}{lcc}
\hline Final diagnosis & Patients $(n=77)$ & Proportion $(\%)$ \\
\hline Breath-holding spells & 13 & 16.9 \\
Gastro-esophageal reflux & 11 & 14.3 \\
Jitteriness & 10 & 13.0 \\
Stereotypies & 10 & 13.0 \\
Sleep myoclonus & 6 & 7.8 \\
Tonic eye deviation & 5 & 6.5 \\
Shuddering & 3 & 3.9 \\
Sleep behavior & 2 & 2.6 \\
Startle reflex & 2 & 2.6 \\
Benign myoclonus & 1 & 1.3 \\
Cyanotic spells & 1 & 1.3 \\
Unclassified & 13 & 16.9
\end{tabular}

Patients with a medical history of a condition such as developmental delay or a neurologic disorder (e.g., perinatal asphyxia, congenital or acquired brain lesion, head trauma, central nervous system infection, or migraine) or a familial history of epilepsy were more likely to have epilepsy than NEPMs (11/34, 32.35\% vs. $12 / 77,15.58 \%, P<0.05$ ) (Table 5). The patients with epilepsy showed a non-significantly higher likelihood of having focal motor symptoms $(15 / 34,44.12 \%$ vs. $21 / 77,27.27 \%, P=0.08)$.

All 111 infants with paroxysmal motor events underwent EEG, and abnormalities were present in 24 patients. Epilepsy patients were significantly more likely than patients with NEPMs to have abnormal inter-ictal EEG (23/34, 67.6\% vs. $1 / 77,1.3 \%, P<$ $0.001)$. Twenty-three epilepsy patients had abnormal EEGs, including focal epileptiform discharges $(n=16,47 \%)$, hypsarrhythmia $(n=6,17.6 \%)$, and other background abnormalities $(n=1$, $2.9 \%)$. Only one of the 77 infants with NEPMs had an abnormal inter-ictal EEG with focal epileptiform discharges $(n=1,1.3 \%)$.

Fifty-five (49\%) of the 111 patients underwent neuroimaging studies, and 17 (31\%) had abnormal findings. Epilepsy patients were more likely than patients with NEPMs to have abnormal 
Table 3. Laboratory findings of the patients $(n=69)$

\begin{tabular}{lccc}
\hline Electrolytes & Epilepsy $(n=33)$ & NEPMs $(n=36)$ & $P$ value \\
\hline Calcium $(\mathrm{mg} / \mathrm{dL})$ & $9.9 \pm 0.3$ & $9.9 \pm 0.5$ & 0.998 \\
Phosphorus $(\mathrm{mg} / \mathrm{dL})$ & $6.0 \pm 0.6$ & $6.2 \pm 1.0$ & 0.199 \\
Glucose $(\mathrm{mg} / \mathrm{dL})$ & $104.6 \pm 17.3$ & $106.0 \pm 25.2$ & 0.782 \\
Sodium $(\mathrm{mmol} / \mathrm{L})$ & $137.5 \pm 2.2$ & $137.2 \pm 2.3$ & 0.592 \\
Potassium $(\mathrm{mmol} / \mathrm{L})$ & $4.6 \pm 0.6$ & $4.6 \pm 0.6$ & 0.559 \\
Chloride $(\mathrm{mmol} / \mathrm{L})$ & $103.4 \pm 9.6$ & $104.8 \pm 3.1$ & 0.419 \\
Ammonia $(\mu \mathrm{mol} / \mathrm{L})$ & $49.7 \pm 24.3$ & $54.6 \pm 17.2$ & 0.430 \\
Lactic acid $(\mathrm{mmol} / \mathrm{L})$ & $2.8 \pm 1.9$ & $2.7 \pm 1.7$ & 0.945 \\
\hline
\end{tabular}

Values are presented as mean \pm standard deviation.

NEPM, non-epileptic paroxysmal motor event.
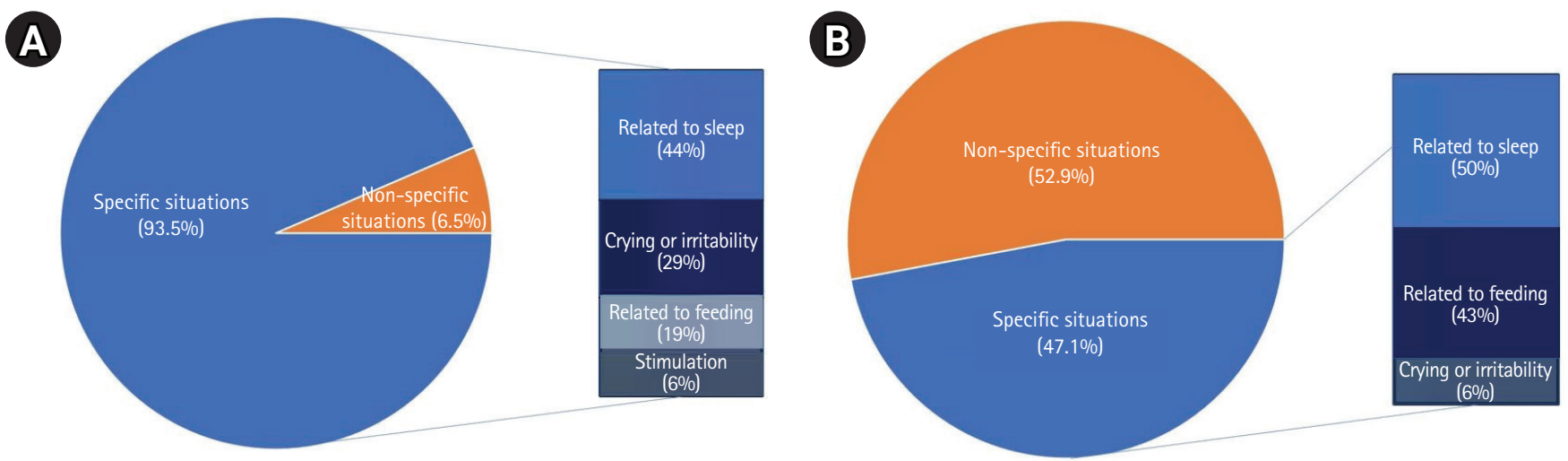

Fig. 1. Clinical characteristics associated with specific situations in (A) non-epileptic paroxysmal motor events and (B) epilepsy.

Table 4. Types and frequencies of specific situations

\begin{tabular}{llc}
\hline Types of specific situations & $\begin{array}{c}\text { Epilepsy } \\
(\mathrm{n}=16)\end{array}$ & $\begin{array}{c}\text { NEPMs } \\
(\mathrm{n}=72)\end{array}$ \\
\hline Related to sleep & $8(50)$ & $32(44)$ \\
Related to feeding & $7(43)$ & $14(19)$ \\
Crying or irritability & $1(6)$ & $21(29)$ \\
Stimulation (suction, bath, defecation, etc.) & 0 & $5(6)$ \\
\hline
\end{tabular}

Values are presented as number (\%).

NEPM, non-epileptic paroxysmal motor event.

brain MRI findings $(13 / 30,43.3 \%$ vs. $4 / 25,16 \%, P=0.02)$ (Table 6).

Univariate and multivariate logistic regression analyses were performed to identify the strongest risk factors of epilepsy. Among the investigated risk factors, medical history $(P=0.049)$, the absence of a specific situation that preceded paroxysmal motor events $(P<0.001)$, focal motor symptoms $(P=0.083)$, and abnormal EEG findings $(P<0.001)$ were significant risk factors for epilepsy in the univariate logistic analysis (Table 5). Among those, clinical risk factors other than EEG were investigated using multivariate logistic analysis to identify independent risk factors of epilepsy (Table 5).

Using the risk factors identified by the multiple logistic regression analysis, a risk scoring system for epilepsy was developed. A score was given to each risk factor using the estimate obtained by penalized maximum likelihood estimates, as shown in Table 7.

Using this scoring system with a total risk score from 0 to 5 points, infants with 2 or more points could be diagnosed with epilepsy with $61.76 \%$ sensitivity and $88.31 \%$ specificity. This cut-off value showed satisfactory discrimination ability, with an area under the curve of 0.814 .

\section{Discussion}

Paroxysmal motor events are a common reason why infants present to pediatric neurology clinics. NEPMs are much more common than epilepsy in these infants, as the cumulative incidence of epilepsy in the first year of life was recently reported to be $0.22 \%$, while that of NEPMs was found to be $0.79 \%$ [12]. Heggstad and Hafstrom [12] reported that $57 \%$ of infants who visited the hospital for paroxysmal motor events had NEPMs, whereas only $17 \%$ were diagnosed with epilepsy. In our study, 77 (69.4\%) of the 111 
Table 5. Univariate and multivariate predictor variables of epilepsy

\begin{tabular}{|c|c|c|c|c|c|c|}
\hline \multirow{2}{*}{ Predictor variable } & \multirow{2}{*}{ Epilepsy } & \multirow{2}{*}{ NEPMs } & \multicolumn{2}{|c|}{ Univariate logistic analysis } & \multicolumn{2}{|c|}{ Multivariate logistic analysis } \\
\hline & & & OR $(95 \% \mathrm{Cl})$ & $P$ value & OR $(95 \% \mathrm{Cl})$ & $P$ value \\
\hline Male sex & $23(67.6)$ & $40(51.9)$ & $1.934(0.83-4.51)$ & 0.127 & NA & NA \\
\hline Gestational age $<37$ weeks & $30(88.2)$ & $64(83.1)$ & $1.523(0.46-5.07)$ & 0.493 & NA & NA \\
\hline \multicolumn{7}{|l|}{ Medical history } \\
\hline Developmental delay & $3(8.8)$ & $3(3.9)$ & NA & NA & NA & NA \\
\hline Neurologic disorder & $5(14.7)$ & $8(10.4)$ & NA & NA & NA & NA \\
\hline Family history of epilepsy & $3(8.8)$ & $1(1.3)$ & NA & NA & NA & NA \\
\hline Total & $11(32.4)$ & $12(15.6)$ & $2.591(1.01-6.67)$ & 0.049 & $2.662(0.87-8.12)$ & 0.086 \\
\hline \multicolumn{7}{|l|}{ Type of motor symptoms } \\
\hline Focal motor symptoms & $15(44.1)$ & $21(27.3)$ & $2.105(0.91-4.89)$ & 0.083 & $2.811(1.02-7.78)$ & 0.047 \\
\hline Generalized motor symptoms & 19 (55.6) & $56(72.7)$ & NA & NA & NA & NA \\
\hline Absence of a specific situation & $18(52.9)$ & $5(6.5)$ & $14.78(4.87-44.89)$ & $<0.001$ & $17.21(5.27-56.2)$ & $<0.001$ \\
\hline Abnormal EEG & $23(67.7)$ & $1(1.3)$ & $104.2(17.29-628.1)$ & $<0.001$ & NA & NA \\
\hline
\end{tabular}

Values are presented as number (\%).

NEPM, non-epileptic paroxysmal motor event; $\mathrm{OR}$, odds ratio; $\mathrm{Cl}$, confidence interval; $\mathrm{NA}$, not applicable; $\mathrm{EEG}$, electroencephalography.

Table 6. Brain MRI abnormalities in patients with epilepsy and NEPMs $(n=17)$

\begin{tabular}{lcc}
\hline Brain MRI abnormalities & Epilepsy $(n=13)$ & NEPMs $(n=4)$ \\
\hline Focal cortical dysplasia & 3 & 0 \\
Ventriculomegaly & 3 & 1 \\
White matter abnormalities & 2 & 0 \\
Periventricular leukomalacia & 1 & 1 \\
Mild-form lobar holoprosencephaly & 1 & 0 \\
Cortical tuber & 1 & 0 \\
Non-specific cystic changes & 1 & 2 \\
Corpus callosum agenesis & 1 & 0
\end{tabular}

$M R I$, magnetic resonance imaging; NEPM, non-epileptic paroxysmal motor event.

Table 7. Risk scoring system for epilepsy diagnosis in infants with paroxysmal motor events

\begin{tabular}{lcrc}
\hline Risk factor & Estimates & $\chi^{2}$ test & Score \\
\hline Medical history & 0.490 & 0.086 & 1 \\
Absence of a specific situation & 1.423 & $<0.001$ & 3 \\
Focal motor symptoms & 0.517 & 0.047 & 1 \\
\hline
\end{tabular}

patients had NEPMs, and the others were diagnosed with epilepsy; this ratio is similar to those reported in other studies.

Epilepsy in infancy not only results in seizures, but can also have profound consequences for neurocognitive development, behavior, and quality of life. In patients with epilepsy, optimal treatment with appropriate use of anti-seizure medications is needed, and an additional etiologic work-up, including brain MRI and genetic/ metabolic studies, is imperative [13]. Unlike epilepsy, most NEPMs disappear without specific treatment. An accurate diagnosis of
NEPMs can reduce the unnecessary use of antiepileptic drugs and the large number of investigations in children with NEPMs. However, the differentiation between epileptic and NEPMs can be very difficult, especially in infancy. Moreover, the highly diverse phenotypes of NEPMs during infancy and ambiguous phenotypic differences make the differential diagnosis of NEPMs more challenging [14].

In light of the obstacles described above, all patients in this study underwent routine EEG to differentiate epileptic seizures from NEPMs. If patients had focal/generalized epileptiform discharges and started anti-seizure medications, we assumed that those patients had focal/generalized epilepsy. One exceptional patient with NEPMs was first reported to have focal epileptiform discharges on routine EEG; however, his events occurred only once and he did not start an anti-seizure medication.

In our study, we demonstrated that three clinical factors-namely, the presence of a history of medical illness, paroxysmal events in the absence of specific situations, and focal motor symptomswere independent risk factors for epilepsy. Although our study did not include it in the multivariate analysis, EEG was also a significant risk factor for epilepsy in the univariate analysis. The reason why EEG was not included in the multivariate analysis was selection bias in our clinical diagnostic paradigm, wherein clinicians tended to diagnose infants as having epilepsy when their routine EEG recording showed epileptiform discharges. For this reason, we excluded EEG findings to develop a screening system that evaluates the risk of epilepsy, and this system showed relatively good sensitivity and specificity.

Several studies have investigated risk factors for epilepsy in infants, such as that by Heggstad and Hafstrom [12]. According to 
their study, abnormal brain MRI findings, abnormal EEG findings, and history of neonatal care increased the likelihood of having epilepsy in infants. In addition, the presence of specific situation-related factors lowered the epilepsy risk, which agrees with the results of our study. Maia et al. [11] identified seizures occurring during sleep, remote symptomatic etiology, and abnormal EEG results as risk factors for recurrence in children with a first seizure episode. Van Diessen et al. [10] reported a prediction model based on clinical characteristics that was useful in diagnosing epilepsy in children with one or more paroxysmal motor events. However, they only included older children, and no previous study has presented an epilepsy prediction model in infants. In other studies $[15,16]$, some authors have reported a scoring system of epilepsy to predict the neurological outcomes or prognosis of epilepsy, but there is no scoring system for epilepsy diagnosis. In this study, eight patients were diagnosed with infantile spasms, which could be a possible confounding factor of the scoring system. Since the diagnostic triad of infantile spasms consists of epileptic spasms in infancy, hypsarrhythmia on EEG, and developmental regression, the semiology of infantile spasms and EEG were not included as risk stratification factors to reduce the impact of infantile spasms on the scoring system.

This study has some limitations. First, it is possible that some demographic and clinical data were lost and under-represented in some cases due to the retrospective nature of this study. Secondly, the relatively small number of patients and the lack of laboratory data, such as brain MRI findings, may be other limitations. Future research with a larger number of infants will allow a more detailed risk factor stratification.

The strength of this study is that it is the first to suggest a scoring system with potential clinical applicability that facilitates the differential diagnosis of epilepsy and NEPMs. Our scoring system does not require any invasive, time-consuming examinations; instead, it uses an informative history that can be acquired from patients' caregivers. Thus, we expect that this simple screening system can help clinicians decide whether numerous epilepsy investigations are necessary. It should be noted that this scoring system had relatively low sensitivity for epilepsy despite its high specificity. Considering the risk-benefit balance of useless treatment and untreated seizure burden in this age group, we chose high specificity over sensitivity for epilepsy.

In conclusion, in infants who presented with paroxysmal motor events, NEPMs were a more common diagnosis than epilepsy. This study showed that specific situations that preceded paroxysmal motor events, a medical history of another illness, and focal motor symptoms might be possible clues for making a diagnosis of epilepsy. These results re-emphasize the significance of taking an informative clinical history from infants' caregivers. Additionally, a newly developed scoring system with these clinical variables might be a good clinical indicator to identify infants at high risk for epilepsy. A further prospective study with a larger number of patients is needed to validate this new scoring system.

\section{Conflicts of interest}

No potential conflict of interest relevant to this article was reported.

\section{ORCID}

Han Jun Kim, https://orcid.org/0000-0002-0173-727X

Mi-Sun Yum, https://orcid.org/0000-0002-5986-5258

\section{Author contribution}

Conceptualization: HJK, HNJ, MSY, HA, and TSK. Data curation: HJK, HNJ, HA, and TSK. Formal analysis: TSK. Funding acquisition: MSY and TSK. Methodology: HNJ, HA, and MSY. Project administration: HNJ and MSY. Visualization: HJK. Writing-original draft: HJK. Writing-review \& editing: HNJ and MSY.

\section{References}

1. Prasad M, Babiker MO. Fifteen-minute consultation: when is a seizure not a seizure? Part 1, the younger child. Arch Dis Child Educ Pract Ed 2016;101:15-20.

2. Babiker MO, Prasad M. Fifteen-minute consultation: when is a seizure not a seizure? Part 2, the older child. Arch Dis Child Educ Pract Ed 2015;100:295-300.

3. Ito Y, Kidokoro H, Negoro T, Tanaka M, Okai Y, Sakaguchi Y, et al. Paroxysmal nonepileptic events in children with epilepsy. Epilepsy Res 2017;132:59-63.

4. Modi AC, King AS, Monahan SR, Koumoutsos JE, Morita DA, Glauser TA. Even a single seizure negatively impacts pediatric health-related quality of life. Epilepsia 2009;50:2110-6.

5. Uldall P, Alving J, Hansen LK, Kibaek M, Buchholt J. The misdiagnosis of epilepsy in children admitted to a tertiary epilepsy centre with paroxysmal events. Arch Dis Child 2006;91:219-21.

6. Hamiwka LD, Singh N, Niosi J, Wirrell EC. Diagnostic inaccuracy in children referred with "first seizure": role for a first seizure clinic. Epilepsia 2007;48:1062-6.

7. DiMario FJ Jr. Paroxysmal nonepileptic events of childhood. Semin Pediatr Neurol 2006;13:208-21.

8. Fusco L, Specchio N. Non-epileptic paroxysmal manifestations 
during sleep in infancy and childhood. Neurol Sci 2005;26 Suppl 3:s205-9.

9. Canavese C, Canafoglia L, Costa C, Zibordi F, Zorzi G, Binelli S, et al. Paroxysmal non-epileptic motor events in childhood: a clinical and video-EEG-polymyographic study. Dev Med Child Neurol 2012;54:334-8.

10. van Diessen E, Lamberink HJ, Otte WM, Doornebal N, Brouwer OF, Jansen FE, et al. A prediction model to determine childhood epilepsy after 1 or more paroxysmal events. Pediatrics 2018;142:e20180931.

11. Maia C, Moreira AR, Lopes T, Martins C. Risk of recurrence after a first unprovoked seizure in children. J Pediatr (Rio J) 2017;93:281-6.

12. Heggstad N, Hafstrom M. Seizure-like events leading to hospital referrals in infants: a retrospective population-based study. Acta Paediatr 2021;110:584-93.

13. Alam S, Lux AL. Epilepsies in infancy. Arch Dis Child 2012; 97:985-92.

14. Jung SY, Kang JW. Is it really a seizure?: the challenge of paroxysmal nonepileptic events in young infants. Clin Exp Pediatr 2021;64:384-92.

15. Kim HJ, Lee BS, Yum MS, Jang HJ, Oh SH, Do HJ, et al. Seizure scoring system and neurodevelopmental outcomes in very low birth weight infants with neonatal seizures. Neonatal Med 2013;20:428-37.

16. Chen X, Ma XB, Zhang Q Yin Q Li XH. A scale for predicting the outcomes of patients with epilepsy: a study of 141 cases. Int J Gen Med 2021;14:1565-74. 